\title{
ASYMPTOTICS OF HYBRID FLUID QUEUES WITH LÉVY INPUT
}

\author{
KRZYSZTOF DȨBICKI*** AND \\ IWONA SIERPIŃSKA, ${ }^{* * * *}$ University of Wroctaw \\ BERT ZWART, ${ }^{* * * *}$ CWI, VU University Amsterdam, EURANDOM \\ and Georgia Institute of Technology
}

\begin{abstract}
Let $\{X(t): t \in \mathbb{R}\}$ be the integrated on-off process with regularly varying on-periods, and let $\{Y(t): t \in \mathbb{R}\}$ be a centered Lévy process with regularly varying positive jumps (independent of $X(\cdot))$. We study the exact asymptotics of $\mathbb{P}\left(\sup _{t \geq 0}\{X(t)+Y(t)-c t\}>u\right)$ as $u \rightarrow \infty$, with special attention to the case $r=c$, where $r$ is the increase rate of the on-off process during the on-periods.
\end{abstract}

Keywords: Exact asymptotics; Lévy process; fluid model; queue

2010 Mathematics Subject Classification: Primary 60G15

Secondary 60G70; 60F10; 60K25

\section{Introduction}

The problem of analyzing the asymptotic behavior of the tail distribution of

$$
\sup _{t \geq 0}\{X(t)+Y(t)-c t\}
$$

where $\{X(t): t \in \mathbb{R}\}$ and $\{Y(t): t \in \mathbb{R}\}$ are independent stochastic processes with stationary increments, $c>\mathbb{E}[X(1)+Y(1)]$, plays an important role in many fields of applied probability. In particular, the steady state buffer content in queues fed by the superposition of two input streams, $X(\cdot)$ and $Y(\cdot)$, has the representation (1.1); see, e.g. [19]. On the other hand, (1.1) can be used to describe the ruin probability for perturbed ruin models; see, e.g. [11] and [22].

Throughout the paper, we assume that $\{X(t): t \in \mathbb{R}\}$ is an integrated on-off process, i.e. during 'on-periods' $X(\cdot)$ increases linearly with rate $r>0$, while it is constant during 'offperiods'. The second process, $Y(\cdot)$, is assumed to be a Lévy motion with regularly varying positive jumps, with index $\alpha$.

A large number of results lead to precise asymptotics for the tail distribution of (1.1). These asymptotics often yield the appealing and important qualitative insight that one of the two processes can be replaced by its mean. Results like these are also obtained for systems with more than two input sources, where typically a nontrivial subset of on-off processes is responsible

\footnotetext{
Received 15 June 2009; revision received 29 June 2012.

* Postal address: Instytut Matematyczyny, University of Wrocław, pl. Grunwaldzki 2/4, 50-384 Wrocław, Poland.

** Email address: krzysztof.debicki@math.uni.wroc.pl

Supported by MNiSW grant N N2014079 33 (2007-2009) and by a Marie Curie Transfer of Knowledge Fellowship of the European Community's Sixth Framework Programme under contract MTKD-CT-2004-013389.

*** Email address: iwona.sierpinska@math.uni.wroc.pl

**** Email address: bert.zwart@cwi.nl

Partly supported by NSF grants 0727400 and 0805979 , an IBM facility award, and a VIDI grant from NWO.
} 
for overflow; cf. [4], [13], and [26]. The first result of this type was proved in Theorem 4.4 of [13], as noted in [1], which termed these results reduced load equivalence. See [12], [14], [20], [24], and [25] for more references.

As in the abovementioned references, the aim of this paper is to derive the exact asymptotics for the tail distribution of (1.1) within the setting of an on-off process and a Lévy process. It turns out that this setting is tractable enough to obtain some tail estimates in a situation where the so-called reduced load equivalence does not hold.

For completeness, we consider the more standard situation as well, which occurs if $r \neq c$. In this case, either the process $X(\cdot)$ or $Y(\cdot)$ can be replaced with its mean, while the other process is entirely responsible for the tail behavior. It is also possible that both scenarios can occur if the tail of the on-period is regularly varying with index $\alpha$ (i.e. the tail of the on-period is as heavy as the tails of the jumps of the Lévy process). Section 3 contains precise statements, and a proof, which utilizes general results in [26].

The main contribution of the present paper is a detailed analysis of the critical case $r=c$, which is analyzed under the additional assumption that $Y(\cdot)$ is $\alpha$-stable, $\alpha \in(1,2)$. This case is not covered by the above references, and as will be shown, it leads to genuinely different behavior. We give a precise mathematical statement in Section 4, and provide a more informal description here. If $r=c$, the process $X(t)+Y(t)-c t$ has the same increments as $Y(t)$ during on-periods. For $Y(\cdot)$ a Brownian motion, Zwart et al. [24] showed that the most likely way for (1.1) to reach level $u$ is by a single big on-period which has already taking place at time 0 ; this on-period should have length of order $u^{2}$. During that on-period, level $u$ is reached by the oscillatory behavior of the Brownian motion.

In the more general $\alpha$-stable case, it is tempting to conjecture that an initial on-period of size $u^{\alpha}$ suffices. If a standard on-period is regularly varying with index $\gamma$, a residual on-period is regularly varying with index $\gamma-1$, so that the probability of a residual on-period exceeding $u^{\alpha}$ is regularly varying with index $\alpha(\gamma-1)$. However, a different way for (1.1) to reach high level $u$ is by a single big jump of the $Y(\cdot)$ process, which will have a probability which is regularly varying with index $\alpha-1$. Comparing $\alpha-1$ with $\alpha(\gamma-1)$ then leads to the critical value $\gamma^{*}=(2 \alpha-1) / \alpha$. It is shown in Section 4 that if $\gamma<\gamma^{*}$, a single big on-period of length $u^{\alpha}$ suffices for (1.1) to happen, and if $\gamma>\gamma^{*},(1.1)$ is most likely caused by a single big jump of the $Y(\cdot)$ process. The way we prove this is by using the regenerative structure of the process $X(\cdot)+Y(\cdot)$, using techniques from [2] and [17].

The paper is organized as follows. In Section 2 we introduce the notation and describe the analyzed model. In Section 3 we analyze the case where $r \neq c$. The more intricate case $r=c$ is analyzed in Section 4.

\section{Notation and model description}

We consider a fluid queue with infinite buffer capacity, fed by the superposition of two independent input processes, $X(\cdot)$ and $Y(\cdot)$, with stationary increments and emptied at a constant rate $c$.

Following Reich's representation [19], under the stability condition $\mathbb{E}[X(1)+Y(1)]<c$, the steady state buffer content $Q_{X+Y}^{c}$ has the representation

$$
Q_{X+Y}^{c} \stackrel{\mathrm{D}}{=} \sup _{t \geq 0}\{X(t)+Y(t)-c t\}
$$

where ' $=$, denotes equality in distribution. 
Before describing $X(\cdot)$ and $Y(\cdot)$, let us introduce some notation. For a nonnegative random variable $X$, by $X^{r}$ we denote the random variable with density $\mathbb{P}(X>t) / \mathbb{E}[X]$. We write $f(x) \sim g(x)$ if $\lim _{x \rightarrow \infty} f(x) / g(x)=1$ and $f(x) \lesssim g(x)$ if $\lim _{\sup _{x \rightarrow \infty}} f(x) / g(x) \leq 1$.

Moreover, for $c>\mathbb{E}[X(1)]$ and $D \subset[0, \infty)$, we define $Q_{X}^{c}(D):=\sup _{t \in D}\{X(t)-c t\}$ and $Q_{X}^{c}:=Q_{X}^{c}([0, \infty))$; we define $Q_{Y}^{c}(D)$ and $Q_{Y}^{c}$ analogously. In order to shorten the notation, let $Q_{Y}^{c}(t):=Q_{Y}^{c}([0, t])$.

We write $X \in \&$ to denote that the distribution of the random variable $X$ belongs to the class of subexponential distributions, and we write $X \in \mathcal{R} \mathcal{V}_{\alpha}$ to denote that the tail distribution of $X$ is regularly varying at $\infty$ with parameter $\alpha$.

Throughout the paper, the process $\{Y(t): t \geq 0\}$ is a zero-mean Lévy motion with heavytailed jumps.

The process $\{X(t): t \geq 0\}$ is an integrated on-off process, that is, $X(t)=r \int_{0}^{t} \eta(s) \mathrm{d} s$, where $\{\eta(t): t \geq 0\}$ is a stationary alternating on-off (0-1) process. Let $\left\{U, U_{i}: i \geq 0\right\}$ be independent and identically distributed (i.i.d.) nonnegative random variables representing onperiods, and let $\left\{V, V_{i}: i \geq 0\right\}$ be i.i.d. nonnegative random variables representing off-periods. We assume that $\left\{U_{i}: i \geq 0\right\}$ and $\left\{V_{i}: i \geq 0\right\}$ are mutually independent, and that $\mathbb{E} U<\infty$ and $\mathbb{E} V<\infty$. We impose the assumption that

(A) $U \in \mathcal{R} \mathcal{V}_{\gamma}$ with $\gamma:=\gamma_{\text {on }}>1$ and $Y(1) \in \mathcal{R} \mathcal{V}_{\alpha}$ with $\alpha>1$.

For future analysis, we recall the construction of $\eta(\cdot)$, following [10] or [24]. Let

$$
p:=\frac{\mathbb{E} U}{\mathbb{E} U+\mathbb{E} V} .
$$

Additionally, let $I$ be an independent random variable such that $\mathbb{P}(I=1)=1-\mathbb{P}(I=0)=p$. We introduce the delayed renewal sequence

$$
\left\{T_{i}, i \geq 0\right\}:=\left\{T_{0}, T_{0}+\sum_{k=1}^{i}\left(U_{k}+V_{k}\right), i \geq 1\right\}
$$

where

$$
T_{0}=I\left(U_{0}^{r}+V_{0}\right)+(1-I) V_{0}^{r}
$$

Then

$$
\eta(t):=I \mathbf{1}_{\left\{t<U_{0}^{r}\right\}}+\sum_{i=0}^{\infty} \mathbf{1}_{\left\{t \in\left[T_{i} ; T_{i}+U_{i+1}\right)\right\}} .
$$

Additionally, we introduce $X^{\star}(t):=r \int_{0}^{t} \eta^{\star}(s) \mathrm{d} s$, where

$$
\eta^{\star}(t):=\sum_{i=0}^{\infty} \mathbf{1}_{\left\{t \in\left[T_{i}-T_{0}, T_{i}-T_{0}+U_{i+1}\right)\right\}}
$$

is the Palm-stationary version of $\eta(\cdot)$ (that is, an on-period starts at time 0 ). We note that $\rho:=\mathbb{E} X(1)=r p$ and assume the stability condition $\rho<c$.

We finally review the asymptotic behavior of $Q_{X}^{c}$ and $Q_{Y}^{c}$.

The case of an on-off process in isolation is analyzed in [12]. This result holds under our assumptions, and actually continues to hold if the residual on-time has a subexponential tail. 
Proposition 2.1. Let $\rho<c<r$. Then

$$
\mathbb{P}\left(Q_{X}^{c}>u\right) \sim(1-p) \frac{\rho}{c-\rho} \mathbb{P}\left(U^{r}>\frac{u}{r-c}\right) \text { as } u \rightarrow \infty .
$$

For general Lévy processes with subexponential jumps, it is possible to obtain the tail behavior of $Q_{Y}^{c}$ in terms of the tail of $Y(1)$ (cf. [9] and [16]). Both of these works investigate the more general framework of subexponential jumps, which is not necessary for this paper; cf. assumption (A). For stable processes, we note that there is an older result, due to Port [18, Theorem 9].

Proposition 2.2. It holds that

$$
\mathbb{P}\left(Q_{Y}^{c}>u\right) \sim \frac{1}{c} \int_{u}^{\infty} \mathbb{P}(Y(1)>y) \text { dy } \text { as } u \rightarrow \infty .
$$

The following result is due to Willekens [23].

Proposition 2.3. It holds that

$$
\mathbb{P}\left(Q_{Y}^{0}(1)>u\right) \sim \mathbb{P}(Y(1)>u) \quad \text { as } u \rightarrow \infty .
$$

The following proposition combines Breiman's theorem (see [5] and [6, Corollary 3.6]) with the findings of Denisov and Zwart [7, Proposition 2.1].

Proposition 2.4. Suppose that $X$ and $Y$ are independent nonnegative random variables such that $\mathbb{P}(X>u)=L(u) u^{-\alpha}$ for some $\alpha>0$ and $L(\cdot)$ slowly varying at $\infty$.

(i) If $\mathbb{E}\left[Y^{\alpha+\varepsilon}\right]<\infty$ for some $\varepsilon>0$ then

$$
\mathbb{P}(X Y>u) \sim \mathbb{E}\left[Y^{\alpha}\right] \mathbb{P}(X>u) \text { as } u \rightarrow \infty .
$$

(ii) If $\mathbb{P}(Y>u)=o(\mathbb{P}(X>u))$ as $u \rightarrow \infty, \mathbb{E}\left[Y^{\alpha}\right]<\infty$, and

$$
\limsup _{x \rightarrow \infty} \sup _{1 \leq y \leq x} \frac{L(y)}{L(x)}<\infty
$$

then

$$
\mathbb{P}(X Y>u) \sim \mathbb{E}\left[Y^{\alpha}\right] \mathbb{P}(X>u) \text { as } u \rightarrow \infty .
$$

\section{Reduced load equivalence}

In this section we consider the relatively simple case where $r$ and $c$ are not equal. Since the results in this section are reminiscent of several other results in the literature (a recent example arising in GPS queues is [15]), we keep the presentation concise. In particular, we will simply apply existing results in the literature on tail asymptotics of the superposition of heavy-tailed input processes, in particular Theorem 5.3 of [26]. In the case $r<c$, this leads to the following theorem.

Theorem 3.1. Assume that (A) holds. If $r<c$ then

$$
\mathbb{P}\left(Q_{X+Y}^{c}>u\right) \sim \mathbb{P}\left(Q_{Y}^{c-\rho}>x\right) .
$$


The intuition is that, since $r<c$, the on-off process cannot cause a big workload on its own, while a single big jump of the Lévy process suffices (cf. the usual intuition behind the large deviations of $Q_{Y}^{c-\rho}$ ).

Proof of Theorem 3.1. To reduce the model discussed here to the framework of [26], we decompose the process $Y(\cdot)$, i.e. we take $Y(\cdot)=Y_{1}(\cdot)+Y_{2}(\cdot)$, with $Y_{1}(\cdot)$ containing all jumps bigger than size 1 , and $Y_{2}(\cdot)$ containing jumps smaller than size 1 (including all downward jumps). The processes are constructed in such a way that their mean is 0 . By [3], $Q_{Y_{2}}^{c}$ has an exponential right tail for all $c>0$, implying that $Y_{2}(\cdot)$ satisfies Assumption 2.1 of [26].

In the terminology of the setup preceding Theorem 5.3 of [26], $X(\cdot)$ is not critical, and, therefore, not dominant. Since $Y_{1}(\cdot)$ is the only process with instantaneous heavy-tailed input, it is also dominant, implying the result.

The case $r>c$ is somewhat richer, since now either the on-off process or the Lévy process, or both can cause the event $\left\{Q_{X+Y}^{c}>u\right\}$ to happen. In terms of input parameters, this corresponds to the cases $\alpha<\gamma, \alpha=\gamma$, and $\alpha>\gamma$.

Theorem 3.2. Assume that (A) holds. Let $r>c$.

(i) If $\alpha<\gamma$ then

$$
\mathbb{P}\left(Q_{X+Y}^{c}>u\right) \sim \mathbb{P}\left(Q_{Y}^{c-\rho}>u\right) .
$$

(ii) If $\alpha>\gamma$ then

$$
\mathbb{P}\left(Q_{X+Y}^{c}>u\right) \sim \mathbb{P}\left(Q_{X}^{c}>u\right)
$$

(iii) If $\alpha=\gamma$ then

$$
\mathbb{P}\left(Q_{X+Y}^{c}>u\right) \sim \mathbb{P}\left(Q_{X}^{c}>u\right)+\mathbb{P}\left(Q_{Y}^{c-\rho}>u\right) .
$$

Proof. In the terminology of Theorem 5.3 of [26], $X(\cdot)$ is critical since $r>c$, and is weakly dominant if $\gamma \leq \alpha$. The process $Y(\cdot)$ is weakly dominant if $\gamma \geq \alpha$. Theorem 5.3 of [26] then implies the result.

\section{The interplay between rare events and zero-critical behavior}

In this section we focus on the case where a single long on-period makes the system behave in a zero-critical manner during a long period of time, i.e. $r=c$. As discussed in the introduction, this case is much less understood. We show that the tail asymptotics in this case are qualitatively different. In fact, the tail behavior looks quite unusual as the following theorem shows. In this section we will also assume that $Y(\cdot)$ is an $\alpha$-stable process, $\alpha \in(1,2)$ and $\beta \in(-1,1]$.

Theorem 4.1. Assume that $(A)$ holds and that $Y(\cdot)$ is an $\alpha$-stable process with $\alpha \in(1,2)$ and $\beta \in(-1,1]$. Let $r=c$.

(i) If $\gamma \in(1,(2 \alpha-1) / \alpha)$ then

$$
\mathbb{P}\left(Q_{X+Y}^{c}>u\right) \sim p \mathbb{E}\left[Q_{Y}^{0}(1)^{\alpha(\gamma-1)}\right] \mathbb{P}\left(U^{r}>u^{\alpha}\right) .
$$

(ii) If $\gamma=(2 \alpha-1) / \alpha$ then

$$
\mathbb{P}\left(Q_{X+Y}^{c}>u\right) \sim p \mathbb{E}\left[Q_{Y}^{0}(1)^{\alpha(\gamma-1)}\right] \mathbb{P}\left(U^{r}>u^{\alpha}\right)+\mathbb{P}\left(Q_{Y}^{c-\rho}>u\right) .
$$

(iii) If $\gamma>(2 \alpha-1) / \alpha$ then

$$
\mathbb{P}\left(Q_{X+Y}^{c}>u\right) \sim \mathbb{P}\left(Q_{Y}^{c-\rho}>u\right)
$$


An informal explanation of this result is given in the introduction. The rest of this section is devoted to the formal proof. The idea of the proof is to use the regenerative structure of the input process $X(\cdot)+Y(\cdot)$. In particular, we choose the beginning of on-periods as regeneration epochs. In Lemmas 4.1-4.3 below we investigate the zero-delayed case where an on-period starts at time 0 . We use ideas from [2] (see also [17]) to show that the tail asymptotics can be reduced to those of the random walk which is obtained by sampling the process at regeneration epochs only. The necessary conditions under which this holds are checked in Lemmas 4.1 and 4.2, after which Lemma 4.3 concludes.

Since our primary interest is the delayed (time-stationary) case, we need to investigate what may happen until the first renewal epoch. This is done in Lemma 4.4 below. The section closes with the proof of Theorem 4.1 .

Lemma 4.1. It holds that

$$
\mathbb{P}\left(Q_{Y}^{c}(V)>u\right) \sim \mathbb{E}[V] \mathbb{P}(Y(1)>u) \text { as } u \rightarrow \infty .
$$

Proof. Upper bound. Observe that, due to the self-similarity of $Y(\cdot)$, we have

$$
Q_{Y}^{c}(V) \leq Q_{Y}^{0}(V) \stackrel{\mathrm{D}}{=} V^{1 / \alpha} Q_{Y}^{0}(1) .
$$

Since $Y(\cdot)$ is $\alpha$-stable with $\alpha \in(1,2)$ and $\beta \in(-1,1]$, then $\mathbb{P}(Y(1)>u) \sim$ (constant) $u^{-\alpha}$ as $u \rightarrow \infty$; see, e.g. Property 1.2.15 of [21]. Thus, following Propositions 2.4(ii) and 2.3, we have

$$
\mathbb{P}\left(Q_{Y}^{c}(V)>u\right) \leq \mathbb{P}\left(V^{1 / \alpha} Q_{Y}^{0}(1)>u\right) \sim \mathbb{E}[V] \mathbb{P}(Y(1)>u) .
$$

Lower bound. For given $k>0$, using Proposition 2.4, we have

$$
\begin{aligned}
\mathbb{P}\left(Q_{Y}^{c}(V)>u\right) & \geq \mathbb{P}(Y(V)>c V+u) \\
& \geq \mathbb{P}\left(V^{1 / \alpha} Y(1)>c k+u ; V \leq k\right) \\
& \sim \mathbb{E}\left[V \mathbf{1}_{\{V \leq k\}}\right] \mathbb{P}(Y(1)>u) \text { as } u \rightarrow \infty
\end{aligned}
$$

Thus, the lower bound follows by passing with $k \rightarrow \infty$.

This completes the proof.

Lemma 4.2. It holds that

$$
\mathbb{P}\left(\sup _{t \leq U+V}\left\{Y(t)-c(t-U)^{+}\right\}>u\right) \sim \mathbb{P}(Y(U+V)-c V>u) \sim \mathbb{E}[U+V] \mathbb{P}(Y(1)>u) .
$$

Proof. Since $\mathbb{P}\left(\sup _{t \leq U+V}\left\{Y(t)-c(t-U)^{+}\right\}>u\right) \geq \mathbb{P}(Y(U+V)-c V>u)$, then it suffices to find an upper bound for $\mathbb{P}\left(\sup _{t \leq U+V}\left\{Y(t)-c(t-U)^{+}\right\}>u\right)$ and a lower bound for $\mathbb{P}(Y(U+V)-c V>u)$ as $u \rightarrow \infty$ that are tight.

Upper bound. Observe that

$$
\begin{aligned}
\mathbb{P}\left(\sup _{t \leq U+V}\left\{Y(t)-c(t-U)^{+}\right\}>u\right) & \leq \mathbb{P}\left(\sup _{t \leq U+V} Y(t)>u\right) \\
& =\mathbb{P}\left((U+V)^{1 / \alpha} \sup _{t \in[0,1]} Y(t)>u\right) \\
& \sim \mathbb{E}[U+V] \mathbb{P}(Y(1)>u)
\end{aligned}
$$

as $u \rightarrow \infty$, where (4.1) is due to Proposition 2.4(ii) combined with Proposition 2.3. 
Lower bound. Since, for each $u$,

$$
\mathbb{P}(Y(U+V)-c V>u) \geq \mathbb{P}(Y(U+V)-c(U+V)>u),
$$

then the lower bound follows by the same argument as in the proof of the lower bound of Lemma 4.1.

Lemma 4.3. Let $c=r$. Then

$$
\mathbb{P}\left(\sup _{t \geq 0}\left\{X^{\star}(t)+Y(t)-c t\right\}>u\right) \sim \mathbb{P}\left(Q_{Y}^{c-\rho}>u\right) .
$$

Proof. The idea of the proof is to check the assumptions of Theorem 3.3 of [2] (see also Theorem 1 of [17]). In particular, it suffices to prove that

$$
\mathbb{P}\left(\sup _{t \leq U+V}\left\{Y(t)-c(t-U)^{+}\right\}>u\right) \sim \mathbb{P}(Y(U+V)-c V>u),
$$

which straightforwardly follows from Lemma 4.2.

We now investigate what happens in the more general delayed case.

Lemma 4.4. (i) If $\gamma \geq 2$ then

$$
\mathbb{P}\left(\sup _{t \leq U^{r}+V}\left\{Y(t)-c\left(t-U^{r}\right)^{+}\right\}>u\right)=o\left(u^{1 / 2-\alpha}\right) .
$$

(ii) If $\gamma \in(1,2)$ then

$$
\mathbb{P}\left(\sup _{t \leq U^{r}+V}\left\{Y(t)-c\left(t-U^{r}\right)^{+}\right\}>u\right) \sim \mathbb{E}\left[Q_{Y}^{0}(1)^{\alpha(\gamma-1)}\right] \mathbb{P}\left(U^{r}>u^{\alpha}\right) \in \mathcal{R} \mathcal{V}_{\alpha(\gamma-1)}
$$

Proof. We distinguish between three cases: $\gamma>2, \gamma=2$, and $\gamma \in(1,2)$.

If $\gamma>2$ then, following the proof of Lemma 4.2, we have

$$
\mathbb{P}\left(\sup _{t \leq U^{r}+V}\left\{Y(t)-c\left(t-U^{r}\right)^{+}\right\}>u\right) \sim \mathbb{E}\left[U^{r}+V\right] \mathbb{P}(Y(1)>u)=o\left(u^{1 / 2-\alpha}\right)
$$

as $u \rightarrow \infty$.

For the remaining cases, note that

$$
\begin{aligned}
& \mathbb{P}\left(\sup _{t \leq U^{r}+V}\left\{Y(t)-c\left(t-U^{r}\right)^{+}\right\}>u\right) \\
& \quad=\mathbb{P}\left(\sup _{t \leq U^{r}} Y(t)>u \text { or } \sup _{U^{r} \leq t \leq U^{r}+V}\left\{Y(t)-c\left(t-U^{r}\right)\right\}>u\right) \\
& \quad=\mathbb{P}\left(\sup _{t \leq U^{r}} Y(t)>u \text { or } Y\left(U^{r}\right)+\sup _{t \leq V}\left\{Y\left(U^{r}+t\right)-Y\left(U^{r}\right)-c t\right\}>u\right) \\
& \quad \leq \mathbb{P}\left(Q_{Y}^{0}\left(U^{r}\right)>u \text { or } Q_{Y}^{0}\left(U^{r}\right)+\sup _{t \leq V}\{\tilde{Y}(t)-c t\}>u\right) \\
& \quad=\mathbb{P}\left(Q_{Y}^{0}\left(U^{r}\right)+Q_{\tilde{Y}}^{c}(V)>u\right),
\end{aligned}
$$

where $\tilde{Y}(\cdot)$ is a stochastically independent copy of $Y(\cdot)$. Let $\gamma=2$. Combining Lemma 4.1 
with the observation that $\left(U^{r}\right)^{1 / \alpha}, Q_{Y}^{0}(1) \in \mathcal{R} \mathcal{V}_{\alpha}$ yields (applying the corollary to Theorem 3 of [8])

$$
Q_{Y}^{0}\left(U^{r}\right) \stackrel{\mathrm{D}}{=}\left(U^{r}\right)^{1 / \alpha} Q_{Y}^{0}(1) \in \mathcal{R} \mathcal{V}_{\alpha}
$$

Hence, due to Lemma 4.1, $\mathbb{P}\left(Q_{Y}^{0}\left(U^{r}\right)+Q_{Y}^{c}(V)>u\right) \in \mathcal{R} \mathcal{V}_{\alpha}$, which completes the proof in the $\gamma=2$ case.

If $\gamma \in(1,2)$ then Proposition 2.4(i) yields

$$
\mathbb{P}\left(Q_{Y}^{0}\left(U^{r}\right)>u\right)=\mathbb{P}\left(\left(U^{r}\right)^{1 / \alpha} Q_{Y}^{0}(1)>u\right) \sim \mathbb{E}\left[Q_{Y}^{0}(1)^{\alpha(\gamma-1)}\right] \mathbb{P}\left(U^{r}>u^{\alpha}\right) \in \mathcal{R} \mathcal{V}_{\alpha(\gamma-1)} .
$$

Thus, (4.2) combined with Lemma 4.1 and the lower bound

$$
\mathbb{P}\left(\sup _{t \leq U^{r}+V}\left\{Y(t)-c\left(t-U^{r}\right)^{+}\right\}>u\right) \geq \mathbb{P}\left(Q_{Y}^{0}\left(U^{r}\right)>u\right)
$$

implies the claimed asymptotics. This completes the proof.

We now complete the proof of Theorem 4.1. Note that there is a similarity with Corollary 3.2 of [2]. However, this corollary would only apply to two of our three cases. In addition, given the overlap between cases, we prefer to give a self-contained proof.

Proof of Theorem 4.1. First, we prove that

$$
\begin{aligned}
\mathbb{P}\left(Q_{X+Y}^{c}>u\right) \sim & p \mathbb{P}\left(\sup _{t \leq U^{r}+V}\left\{Y(t)-c\left(t-U^{r}\right)^{+}\right\}>u\right) \\
& +\mathbb{P}\left(\sup _{t \geq 0}\left\{X^{*}(t)+Y(t)-c t\right\}>u\right) .
\end{aligned}
$$

Following the construction of process $X(t)$ (see Section 2), we have

$$
\mathbb{P}\left(Q_{X+Y}^{c}>u\right)=\mathbb{P}\left(\sup _{t \leq T_{0}}\{X(t)+Y(t)-c t\}>u \text { or } \sup _{t \geq T_{0}}\{X(t)+Y(t)-c t\}>u\right)
$$

with

$$
\begin{aligned}
\mathbb{P}\left(\sup _{t \leq T_{0}}\{X(t)+Y(t)-c t\}>u\right)= & p \mathbb{P}\left(\sup _{t \leq U^{r}+V}\left\{Y(t)-c\left(t-U^{r}\right)^{+}\right\}>u\right) \\
& +(1-p) \mathbb{P}\left(Q_{Y}^{c}\left(V^{r}\right)>u\right) .
\end{aligned}
$$

Moreover,

$$
\mathbb{P}\left(Q_{Y}^{c}\left(V^{r}\right)>u\right) \leq \mathbb{P}\left(\sup _{t \leq u^{1 / 2}} Y(t)>u\right)+\mathbb{P}\left(V^{r}>u^{1 / 2}\right) \mathbb{P}\left(\sup _{t \geq 0}\{Y(t)-c t\}>u\right) .
$$

Since $Y(\cdot)$ is self-similar, then $\sup _{t \leq u} 1 / 2 Y(t) \in \mathcal{R} \mathcal{V}_{\alpha-1 / 2}$. Together with Proposition 2.2, Proposition 2.3, and Lemma 4.3, this implies that

$$
\mathbb{P}\left(Q_{Y}^{c}\left(V^{r}\right)>u\right)=o\left(\mathbb{P}\left(\sup _{t \geq 0}\left\{X^{*}(t)+Y(t)-c t\right\}>u\right)\right) \quad \text { as } u \rightarrow \infty .
$$


Upper bound. In order to get the asymptotic upper bound in (4.3), we majorize (4.4) by

$$
\begin{aligned}
& \mathbb{P}\left(\sup _{t \leq T_{0}}\{X(t)+Y(t)-c t\}+\sup _{t \geq 0}\left\{\tilde{X}^{*}(t)+\tilde{Y}(t)-c t\right\}>u\right) \\
&= p \mathbb{P}\left(\sup _{t \leq U^{r}+V}\left\{Y(t)-c\left(t-U^{r}\right)^{+}\right\}+\sup _{t \geq 0}\left\{\tilde{X}^{*}(t)+\tilde{Y}(t)-c t\right\}>u\right) \\
&+(1-p) \mathbb{P}\left(Q_{Y}^{c}\left(V^{r}\right)+\sup _{t \geq 0}\left\{\tilde{X}^{*}(t)+\tilde{Y}(t)-c t\right\}>u\right),
\end{aligned}
$$

where $\tilde{X}^{*}(\cdot)$ and $\tilde{Y}(\cdot)$ are stochastically independent copies of $X^{*}(\cdot)$ and $Y(\cdot)$, respectively. Due to (4.6),

$$
\mathbb{P}\left(Q_{Y}^{c}\left(V^{r}\right)+\sup _{t \geq 0}\left\{\tilde{X}^{*}(t)+\tilde{Y}(t)-c t\right\}>u\right) \sim \mathbb{P}\left(\sup _{t \geq 0}\left\{\tilde{X}^{*}(t)+\tilde{Y}(t)-c t\right\}>u\right),
$$

and following Lemmas 4.3 and 4.4,

$$
\begin{aligned}
& \mathbb{P}\left(\sup _{t \leq U^{r}+V}\left\{Y(t)-c\left(t-U^{r}\right)^{+}\right\}+\sup _{t \geq 0}\left\{\tilde{X}^{*}(t)+\tilde{Y}(t)-c t\right\}>u\right) \\
& \quad \sim \mathbb{P}\left(\sup _{t \leq U^{r}+V}\left\{Y(t)-c\left(t-U^{r}\right)^{+}\right\}>u\right)+\mathbb{P}\left(\sup _{t \geq 0}\left\{\tilde{X}^{*}(t)+\tilde{Y}(t)-c t\right\}>u\right) .
\end{aligned}
$$

This yields the asymptotic upper bound

$$
\mathbb{P}\left(Q_{X+Y}^{c}>u\right) \lesssim p \mathbb{P}\left(\sup _{t \leq U^{r}+V}\left\{Y(t)-c\left(t-U^{r}\right)^{+}\right\}>u\right)+\mathbb{P}\left(\sup _{t \geq 0}\left\{X^{*}(t)+Y(t)-c t\right\}>u\right) .
$$

Lower bound. Let $y>0$ be given. Then (4.4) can be minorized by

$$
\begin{aligned}
& \mathbb{P}\left(\sup _{t \leq T_{0}}\{X(t)+Y(t)-c t\}>u\right) \\
& +\mathbb{P}\left(\left(X\left(T_{0}\right)+Y\left(T_{0}\right)-c T_{0}\right) \in(-y, y),\right. \\
& \left.\quad\left(X\left(T_{0}\right)+Y\left(T_{0}\right)-c T_{0}\right)+\sup _{t>T_{0}}\left\{X(t)+Y(t)-c t-X\left(T_{0}\right)-Y\left(T_{0}\right)+c T_{0}\right\}>u\right) \\
& -\mathbb{P}\left(\sup _{t \leq T_{0}}\{X(t)+Y(t)-c t\}>u,\left(X\left(T_{0}\right)+Y\left(T_{0}\right)-c T_{0}\right) \in(-y, y),\right. \\
& \left.\quad\left(X\left(T_{0}\right)+Y\left(T_{0}\right)-c T_{0}\right)+\sup _{t>T_{0}}\left\{X(t)+Y(t)-c t-X\left(T_{0}\right)-Y\left(T_{0}\right)+c T_{0}\right\}>u\right) \\
& \quad=P_{1}+P_{2}-P_{3} .
\end{aligned}
$$

Now it suffices to note that

$$
P_{2} \geq \mathbb{P}\left(\left(X\left(T_{0}\right)+Y\left(T_{0}\right)-c T_{0}\right) \in(-y, y)\right) \mathbb{P}\left(\sup _{t \geq 0}\left\{X^{*}(t)+Y(t)-c t\right\}>u+y\right),
$$

which implies that

$$
\liminf _{y \rightarrow \infty} \lim _{u \rightarrow \infty} \frac{P_{2}}{\mathbb{P}\left(\sup _{t \geq 0}\left\{X^{*}(t)+Y(t)-c t\right\}>u\right)} \geq 1
$$


and

$$
\begin{aligned}
P_{3} & \leq \mathbb{P}\left(\sup _{t \leq T_{0}}\{X(t)+Y(t)-c t\}>u\right) \mathbb{P}\left(\sup _{t \geq 0}\left\{X^{*}(t)+Y(t)-c t\right\}>u-y\right) \\
& \sim \mathbb{P}\left(\sup _{t \leq T_{0}}\{X(t)+Y(t)-c t\}>u\right) \mathbb{P}\left(\sup _{t \geq 0}\left\{X^{*}(t)+Y(t)-c t\right\}>u\right) \\
& =o\left(\min \left(\mathbb{P}\left(\sup _{t \leq T_{0}}\{X(t)+Y(t)-c t\}>u\right), \mathbb{P}\left(\sup _{t \geq 0}\left\{X^{*}(t)+Y(t)-c t\right\}>u\right)\right)\right)
\end{aligned}
$$

as $u \rightarrow \infty$. Application of the above to (4.8) yields the asymptotic lower bound

$$
\begin{aligned}
\mathbb{P}\left(Q_{X+Y}^{c}>u\right) \gtrsim & \mathbb{P}\left(\sup _{t \leq T_{0}}\{X(t)+Y(t)-c t\}>u\right)+\mathbb{P}\left(\sup _{t \geq 0}\left\{X^{*}(t)+Y(t)-c t\right\}>u\right) \\
= & p \mathbb{P}\left(\sup _{t \leq U^{r}+V}\left\{Y(t)-c\left(t-U^{r}\right)^{+}\right\}>u\right)+(1-p) \mathbb{P}\left(Q_{Y}^{c}\left(V^{r}\right)>u\right) \\
& +\mathbb{P}\left(\sup _{t \geq 0}\left\{X^{*}(t)+Y(t)-c t\right\}>u\right) \\
\sim & p \mathbb{P}\left(\sup _{t \leq U^{r}+V}\left\{Y(t)-c\left(t-U^{r}\right)^{+}\right\}>u\right) \\
& +\mathbb{P}\left(\sup _{t \geq 0}\left\{X^{*}(t)+Y(t)-c t\right\}>u\right),
\end{aligned}
$$

where (4.9) is due to (4.6). This completes the proof of (4.3). Now it suffices to note that, due to Lemmas 4.4 and 4.3 we can distinguish three cases.

- If $\gamma \in(1,(2 \alpha-1) / \alpha)$ then

$$
\mathbb{P}\left(\sup _{t \leq U^{r}+V}\left\{Y(t)-c\left(t-U^{r}\right)^{+}\right\}>u\right) \sim \mathbb{E}\left[Q_{Y}^{0}(1)^{\alpha(\gamma-1)}\right] \mathbb{P}\left(U^{r}>u^{\alpha}\right)
$$

and

$$
\mathbb{P}\left(\sup _{t \geq 0}\left\{X^{*}(t)+Y(t)-c t\right\}>u\right)=o\left(\mathbb{P}\left(U^{r}>u^{\alpha}\right)\right) .
$$

- If $\gamma=(2 \alpha-1) / \alpha$ then

$$
\sup _{t \leq U^{r}+V}\left\{Y(t)-c\left(t-U^{r}\right)^{+}\right\}, \sup _{t \geq 0}\left\{X^{*}(t)+Y(t)-c t\right\} \in \mathcal{R} \mathcal{V}_{\alpha-1} .
$$

- If $\gamma>(2 \alpha-1) / \alpha$ then

$$
\mathbb{P}\left(\sup _{t \leq U^{r}+V}\left\{Y(t)-c\left(t-U^{r}\right)^{+}\right\}>u\right)=o\left(\mathbb{P}\left(\sup _{t \geq 0}\left\{X^{*}(t)+Y(t)-c t\right\}>u\right)\right) .
$$

This completes the proof.

\section{References}

[1] Agrawal, R., Makowski, A. And Nain, P. (1999). On a reduced load equivalence for fluid queues under subexponentiality. Queueing Systems 33, 5-41.

[2] Asmussen, S., Schmidli, H. And Schmidt, V. (1999). Tail probability for non-standard risk and queueing processes with subexponential jumps. Adv. Appl. Prob. 31, $422-447$.

[3] Bertoin, J. and Doney, R. A. (1994). Cramer's estimate for Lévy processes. Statist. Prob. Lett. 21, $363-365$. 
[4] Borst, S. And Zwart, B. (2005). Fluid queues with heavy-tailed $M / G / \infty$ input. Math. Operat. Res. 30, 852-879.

[5] Breiman, L. (1965). On some limit theorems similar to the arc-sin law. Theory Prob. Appl. 10, 323-331.

[6] Cline, D. B. H. and Samorodnitsky, G. (1994). Subexponentiality of the product of independent random variables. Stoch. Process. Appl. 49, 75-98.

[7] Denisov, D. And Zwart, B. (2007) On a theorem of Breiman and a class of random difference equations. J. Appl. Prob. 44, 1031-1046.

[8] Embrechts, P. And Goldie, C. M. (1980). On closure and factorization properties of subexponential and related distributions. J. Austral. Math. Soc. Ser. A 29, 243-256.

[9] Foss, S., Konstantopoulos, T. and Zachary, S. (2007). Discrete and continuous time modulated random walks with heavy-tailed increments. J. Theoret. Prob. 20, 581-612.

[10] Heath, D., Resnick, S. and Samorodnitsky, G. (1998). Heavy tails and long range dependence in ON/OFF processes and associated fluid models. Math. Operat. Res. 23, 145-165.

[11] Huzak, M., Perman, M., S̆ikić, H. and VondraceK, Z. (2004). Ruin probabilities and decompositions for general perturbed risk processes. Ann. Appl. Prob. 14, 1378-1397.

[12] Jelenković, P. R. and Lazar, A. A. (1999). Asymptotic results for multiplexing subexponential on-off processes. Adv. Appl. Prob. 31, 394-421.

[13] Jelenković, P. And Momčilović, P. (2003). Asymptotic loss probability in a finite buffer fluid queue with heterogeneous heavy-tailed on-off processes. Ann. Appl. Prob. 13, 576-603.

[14] Jelenković, P., MomčIlović, P. And Zwart, B. (2004). Reduced load equivalence under subexponentiality. Queueing Systems 46, 97-112.

[15] Lelarge, M. (2009). Asymptotic behavior of generalized processor sharing queues under subexponential assumptions. Queueing Systems 62, 51-73.

[16] Maulik, K. AND Zwart, B. (2006). Tail asymptotics for exponential functionals of Levy processes. Stoch. Process. Appl. 116, 156-177.

[17] Palmowski, Z. ANd Zwart, B. (2007). Tail asymptotics of the supremum of a regenerative process. J. Appl. Prob. 44, 349-365.

[18] Port, S. C. (1989). Stable processes with drift on the line. Trans. Amer. Math. Soc. 313, 805-841.

[19] Reich, E. (1958). On the integrodifferential equation of Takács. I. Ann. Math. Statist. 29, 563-570.

[20] Rolski, T., Schlegel, S. AND Schmidt, V. (1999). Asymptotics of Palm-stationary buffer content distributions in fluid flow queues. Adv. Appl. Prob. 31, 235-253.

[21] Samorodnitsky, G. And Taqqu, M. S. (1994). Stable Non-Gaussian Random Processes. Chapman and Hall, New York.

[22] Schmidli, H. (1999). Perturbed risk processes: a review. Theory Stoch. Process. 5, 145-165.

[23] Willekens, E. (1987). On the supremum of an infinitely divisible process. Stoch. Process. Appl. 26, $173-175$.

[24] Zwart, B., Borst, S. ANd DȨBICKI, K. (2005). Subexponential asymptotics of hybrid fluid and ruin models. Ann. Appl. Prob. 15, 500-517.

[25] Zwart, B., Borst, S. AND DȨBICKI, K. (2005). Reduced load equivalence for Gaussian processes. Operat. Res. Lett. 33, 502-510.

[26] Zwart, B., Borst, S. And Mandjes, M. (2004). Exact asymptotics for fluid queues fed by heavy-tailed on-off flows. Ann. Appl. Prob. 14, 903-957. 